\title{
Alteraciones Hematológicas y Zootécnicas del Pollo de Engorde bajo Estrés Calórico
}

\author{
Elvis A. Díaz ${ }^{(1)^{\star}}$, William Narváez-Solarte ${ }^{(2)}$ y John A. Giraldo(3) \\ (1) Grupo de Investigación en Nutrición, Metabolismo y Seguridad Alimentaria, Facultad de Ciencias \\ Agropecuarias, Departamento de Producción Agropecuaria, Universidad de Caldas, Manizales, Colombia. \\ (e-mail: elvis.diaz@ucaldas.edu.co) \\ (2) Grupo de Investigación en Nutrición, Metabolismo y Seguridad Alimentaria, Facultad de Ciencias \\ Agropecuarias, Departamento de Salud Animal, Universidad de Caldas, Manizales, Colombia. \\ (3) Grupo de Investigación en Nutrición, Metabolismo y Seguridad Alimentaria, Facultad de Ciencias \\ Agropecuarias, Manizales, Colombia. \\ ${ }^{*}$ Autor a quien debe de ir dirigida la correspondencia.
}

Recibido Sep. 28, 2015; Aceptado Nov. 23, 2015; Versión final Feb. 2, 2016, Publicado Jun. 2016

\begin{abstract}
Resumen
El objetivo de este trabajo fue determinar los cambios hematológicos y zootécnicos del pollo de engorde criado bajo estrés calórico crónico inducido y en las condiciones de temperatura ambiental del Piedemonte Amazónico colombiano. Se utilizaron 500 pollos machos, de dos líneas comerciales, de 21 días de edad, distribuidos en un diseño en bloques completamente aleatorizados, en un esquema factorial $2 \times 2$ (dos líneas de pollo y dos condiciones de temperatura), cinco repeticiones por tratamiento y 25 animales por unidad experimental. Se evaluó recuento leucocitario, leucograma diferencial, hemoglobina, relación heterofilo/linfocito, hematocrito, consumo, ganancia de peso, conversión alimenticia y mortalidad. El factor línea no tuvo efecto sobre las variables evaluadas $(p>0,05)$. Existió diferencia $(p<0,05)$ en la mayoría de las variables evaluadas al ser comparados los indicadores hemáticos y productivos. Se concluyó que la hematología y las variables productivas sufrieron cambios significativos bajo ambas temperaturas experimentales, con mayor afectación de los pollos bajo estrés calórico crónico. El leucograma y las variables productivas se constituyeron como los más sensibles y constantes indicadores de la condición fisiológica de estrés por calor.
\end{abstract}

Palabras clave: pollo de engorde; hematocrito; leucograma; avicultura

\section{Hematologic and Zootechnical Alterations in Broiler under Heat Stress}

\begin{abstract}
The objective of this work was to determine hematologic and zootechnical changes in broiler breeders raised under induced chronic heat stress in the Amazon foothills at room temperature conditions. Five hundred male chickens from two different production lines were used, 21 days old each. They were randomly distributed in a block design with a factorial scheme 2x2 (two chicken production lines and two conditions of temperature), five repetitions per treatment and 25 animals per experimental unit. The following factors were determined: White blood cell count, differential complete blood count, hemoglobin, heterophils/lymphocytes relation, hematocrit, intake, weight gain, feed conversion and mortality. The production line factor did not have any effect over the variables that were evaluated $(p>0,05)$. There was a meaningful difference $(p<0,05)$ in most of the evaluated variables when they were compared to the hematic and productive indicators. In conclusion, that hematology and productive variables suffered significant changes under both experimental temperatures, with more detriment in the broilers under chronic heat stress. The leukogram and the productive variables became the most sensitive and constant indicators in the physiological condition of stress by heat.
\end{abstract}

Keywords: broiler breeders; hematocrit; leukogram; poultry production 


\section{INTRODUCCIÓN}

La avicultura en Colombia se ha convertido en una de las actividades pecuarias de mayor crecimiento durante la última década, evidenciado en parte, por el alto número de aves producidas y beneficiadas. El pollo de engorde criado bajo un sistema comercial, es uno de los animales que expresa mayor eficiencia nutricional y productiva, características que se reflejan en la rápida ganancia de peso. Sin lugar a dudas, la carne de pollo contribuye a la seguridad alimentaria de las poblaciones humanas, ofreciendo a los consumidores una fuente de alimento con excelente calidad nutricional, a un precio asequible (Almeida et al., 2012). La producción de pollo a nivel industrial se realiza bajo confinamiento, manejo que no permite a las aves un alto margen de maniobra, para realizar los ajustes comportamentales necesarios para el mantenimiento de la homeostasis térmica (Amaral et al., 2011). En América Latina los factores climáticos ambientes son pobremente manipulados para obtener un mayor bienestar y producción de las aves, no siendo siempre compatible con las necesidades fisiológicas de los animales (Furlan et al., 2004).

Los cambios ambientales de temperatura dentro de los galpones de producción, pueden generar efectos beneficiosos o perjudiciales sobre los pollos de engorde. Las altas humedades y temperaturas ambientales generadoras de una alta sensación térmica, originan una condición fisiológica en el ave que recibe el nombre de estrés calórico, la cual afecta negativamente la eficiencia productiva y demás variables zootécnicas, inicialmente al deprimir el consumo de alimento (Farfán et al., 2010). Entre tanto, las bajas temperaturas incrementan el consumo de la ración, pero a expensas de una alta conversión alimenticia. La producción intensiva bajo condiciones ambientales que sobrepasan la zona de confort térmico y los límites de termorregulación del pollo de engorde, predisponen a los animales al estrés calórico, al superar su capacidad termorreguladora. Este síndrome de desequilibrio orgánico, induce una serie de mecanismos defensivos, en donde la respuesta orgánica inicial se halla integrada coordinadamente por los sistemas: nervioso, endocrino e inmunológico; promoviendo profundas modificaciones metabólicas, hematofisiológicas (Silva, 2006) y perjudicando la salud de las aves.

Colombia es un país bioclimatológicamente diverso por su ubicación en la Zona Tórrida. La radiación solar y la sensación térmica son relativamente constantes a lo largo del año, aumentando su temperatura promedio en las épocas de verano. Entre estas zonas se encuentra el Piedemonte Amazónico, caracterizada por tener clima cálido, alta precipitación anual y alta humedad relativa, factores estos que aunados, se convierten en factores limitantes para que el pollo de engorde exprese a plenitud su potencial genético (López et al., 2014), porque la presión de selección genética, para obtener rápido crecimiento, dispone al animal a una disminución en la respuesta de anticuerpos mediada por células y aumento a las respuestas inflamatorias, acompañada de un incremento en la producción de calor endógeno, ocasionando susceptibilidad a padecimientos metabólicos y problemas de adaptación a factores ambientales, entre ellos a las temperaturas altas (Lu y Zhang, 2007). No obstante, los linajes de rápido crecimiento, difieren entre sí a la tolerancia a las temperaturas elevadas y a la resistencia al estrés calórico, manifestándolo en diferencias de crecimiento y supervivencia (Cahaner et al., 2008; Silva, 2006; López et al., 2014).

Los indicadores frecuentemente utilizados por los investigadores para la evaluación del estrés en las aves durante la etapa productiva son: los cambios en las respuestas fisiológicas del animal, la interpretación de hemogramas y algunas variables zootécnicas que reflejan el estado interno del animal. No obstante, el valor descriptivo y predictivo de estos índices dependen de las características del estresor, de la respuesta del agente estresado y del contexto en que el estrés ocurre (López et al., 2014).

Esta investigación tuvo por objetivo evaluar en dos líneas genéticas de pollo de engorde, el efecto del estrés calórico crónico inducido y el estrés calórico bajo condiciones ambientales del Piedemonte Amazónico colombiano, sobre la hematología y rendimiento productivo de las aves durante la fase comprendida entre los 21 y 42 días de edad.

\section{MATERIALES Y MÉTODOS}

Localización. El experimento se realizó en la granja "Pasarela" ubicada en el área rural del municipio de Florencia, Caquetá, Colombia; en la zona de vida bosque muy húmedo tropical (BmhT) (Holdridge, 1978), a $1^{\circ} 34^{\prime} 35,58^{\prime \prime}$ de Latitud Norte, $75^{\circ} 32^{\prime} 39,68^{\prime \prime}$ de Longitud Occidental, altitud de 243 msnm y precipitación anual de $3600 \mathrm{~mm}^{3}$. Para el bioensayo, las condiciones ambientales de presión atmosférica, temperatura y humedad relativa se establecieron utilizando un barómetro tipo higrotérmico (PCE-THB 38, exactitud: $0,01 \mathrm{~mm} \mathrm{Hg}$, España 2010), con 1500 mediciones durante diferentes épocas del año (Invierno, transición y verano), en seis lugares de la granja, estableciendo los promedios en $24514,6 \pm 2,12 \mathrm{kpa}, 27 \pm 3,33^{\circ} \mathrm{C}$ y $85 \pm 12,30 \%$. Las aves durante el período experimental recibieron las prácticas de manejo convencionales, en cuanto a planes sanitarios, nutricionales y de alimentación requeridos para cada etapa productiva. Los pollos fueron criados durante las tres primeras semanas de vida, recibiendo el mismo manejo y sometidos a una temperatura inicial de $32^{\circ} \mathrm{C} \pm 2^{\circ} \mathrm{C}$ y una humedad relativa de $66 \pm 1,9 \%$, hasta alcanzar una temperatura ambiente $27,07 \pm 0,5^{\circ} \mathrm{C}$ y una humedad relativa de $78,2 \pm 1,6 \%$ a los 21 días de edad. 
Animales. Fueron utilizados 250 pollos machos de la línea A (COBB 500) y 250 pollos machos de la línea B (ROSS 308), de 21 días de edad. De cada línea genética se formaron grupos homogéneos con un peso promedio inicial de $773 \pm 8 \mathrm{~g}$ los cuales se alojaron en los corrales experimentales.

Etapa experimental. 125 aves de la línea $A$ y 125 de la B, se ubicaron en corrales de 2,5x1,5m, a una densidad de 25 aves por corral, en un galpón "tipo abierto" sometidas a la temperatura ambiental del Piedemonte Amazónico; y similar número de aves de cada linaje y por corral se distribuyeron en otro galpón de ambiente controlado, utilizando dos equipos acondicionadores de aire evaporativo (AS-T190206-Sumo GO ${ }^{\circledR}$, USA 2010). Cada unidad experimental fue provista de un comedero tipo tolva y un bebedero automático tipo campana.

Tratamientos. Los tratamientos se distribuyeron en un diseño en bloques completamente aleatorizados, en un esquema factorial $2 \times 2$ (dos líneas de aves y dos condiciones de temperatura, estrés calórico crónico inducido y bajo condiciones ambientales del Piedemonte Amazónico colombiano), con cinco repeticiones por tratamiento y 25 animales por unidad experimental.

Los tratamientos aplicados desde los 21 hasta los 42 días de edad, consistieron en evaluar al pollo de engorde macho de las dos líneas comerciales bajo las condiciones de estrés calórico crónico, manteniendo constante la temperatura a $30 \pm 1^{\circ} \mathrm{C}$ en un galpón con ambiente controlado y en un galpón "tipo abierto" bajo la temperatura ambiental media del Piedemonte Amazónico. El registro de la temperatura ambiental y la humedad relativa de cada ambiente se realizó diariamente cada dos horas, utilizando termohigrómetros digitales de máximas y mínimas (Kex Germany industries Inc. Modelo $\mathrm{SH}-121$, con sensibilidad de $0,1^{\circ} \mathrm{C}$ y $0,1 \%$, Rothenberg, Alemania 2010), localizado a nivel de la altura de las aves. Las medidas de los promedios de temperatura y humedad relativa ambiental dentro del galpón de los tratamientos con estrés calórico crónico inducido, durante el período experimental fueron de $30,04 \pm 0,77^{\circ} \mathrm{C}$ y $77,66 \pm 13,66 \%$, respectivamente; mientras que bajo las condiciones de temperatura ambiental del Piedemonte Amazónico fueron, correspondientemente, de $29,87 \pm 7,51^{\circ} \mathrm{C}, 82,14 \pm 11,23$.

El programa de iluminación durante el período experimental para todos los tratamientos fue de 20 horas luz día, complementando la luz natural con luz artificial incandescente con una intensidad lumínica media de $10 \pm 0,5$ lux (Abbas et al., 2008).

Las aves estuvieron alimentadas con una dieta a base de maíz, torta de soya y aceite vegetal con $3.100 \mathrm{Kcal}$ de EM y 19,5\% de proteína, según las exigencias nutricionales, descritas por Rostagno et al., (2011) y NRC (1994), para satisfacer los requerimientos nutricionales de las aves durante la fase de engorde (Tabla 1). La dieta fue ofrecida en tipo migaja y al igual que el agua fue consumida a voluntad por las aves, durante todo el período experimental.

En la Tabla 1, Premezcla vitamínica (Contenido por kg/ración): Vit. A. 10.000 UI; Vit. D3 3.000 UI; Vit. E 60 mg; Vit. K 3 mg; Vit. B1 3 mg; Vit. B2 8 mg; Vit. B6 4 mg; Vit. B12 0,014 mg; Ácido Pantoténico 20 mg; Niacina $50 \mathrm{mg}$; Ácido Fólico $2 \mathrm{mg}$; Biotina 0,15 mg. ${ }^{2}$ Premezcla mineral (Contenido por $\mathrm{kg}$ de alimento): $\mathrm{Fe} 40 \mathrm{mg}$; Zn 80 mg; Mn 80 mg; Cu 10 mg; 10.7 mg; Se 0,3 mg.

Muestreo. A los 42 días de edad, después de estar las aves en un período de ayuno de ocho horas, a través de punción de la vena braquial, se obtuvieron cinco muestras, de $8 \mathrm{~mL}$ de sangre/ave, de cada unidad experimental para un total de 25 muestras por tratamiento, para determinar la concentración de hemoglobina, porcentaje de hematocrito, conteo de leucocitos totales y leucograma diferencial. El procedimiento de captura y sangrado no superó el minuto, para evitar el estrés inducido por la manipulación durante la colecta de sangre (Voslarova et al., 2008).

Hematocrito. Fue medido por el método de microhematocrito por centrifugación, utilizándose una microcentrífuga con capilar a 14000 RPM expresado en unidades de porcentaje, (Mitchell y Johns, 2008).

Concentración de hemoglobina. Se obtuvo usando el método de la cianometahemoglobina descrito por Briones et al., (2009).

Conteo de leucocitos Totales. Se determinó utilizando un hemocitómetro Neubauer (Marienfeld, Badén Württemberg, Alemania), utilizando la metodología descrita por Natt y Herrick (1952).

Leucograma diferencial. Se realizó a través del análisis de frotis sanguíneo, teñido con coloración de Wright, contando un total de 200 células cuyo resultado se reportó en porcentaje (Talebi et al., 2005); con determinación de la relación heterofilos/linfocitos (H/L).

Ganancia de peso acumulada. Para su medición se pesaron los pollos a las seis de la mañana antes de ser alimentados. Se estableció por la diferencia entre el peso del ave al día 42 y el peso al día 21. 
Consumo de alimento acumulado. Este se determinó por la diferencia entre el peso del alimento ofrecido y las sobras después de su consumo.

Conversión alimenticia. La conversión alimenticia acumulada se determinó por la relación entre el alimento total consumido y la ganancia de peso durante el período experimental.

Mortalidad. La mortalidad acumulada se obtuvo al dividir el número de pollos muertos en cada unidad experimental durante los 21 días de experimentación y el número de pollos iniciales, expresado en unidades de porcentaje.

Análisis estadístico. Los resultados de las variables evaluadas fueron sometidos a análisis de varianza, utilizando el procedimiento General Linear Models (GLM) de SAS-8.02 (2007). Las diferencias entre medias de tratamientos fueron establecidas mediante la prueba de $\mathrm{F}$ o por la prueba de comparación múltiple de medias de Tukey a un nivel de significancia de $p<0,05$.

Tabla 1: Composición de la dieta experimental para pollos de engorde de 21 a 42 días

\begin{tabular}{|l|c|}
\hline \multicolumn{1}{|c|}{ Ingrediente } & $\%$ \\
\hline Maíz & 64,1 \\
\hline Torta de soya 46\% & 29,5 \\
\hline Aceite vegetal & 2,4 \\
\hline Carbonato de calcio & 1,47 \\
\hline Fosfato bicálcico & 1,63 \\
\hline Sal & 0,46 \\
\hline Premezcla Vitamínica ${ }^{1}$ & 0,05 \\
\hline Premezcla mineral & 0,10 \\
\hline Metionina HA & 0,26 \\
\hline Colina & 0,03 \\
\hline & Niveles nutricionales \\
\hline EM (kcal/kg) & 3.100 \\
\hline PB (\%) & 19,5 \\
\hline Lisina (\%) & 1,14 \\
\hline Metionina+cistina (\%) & 0,83 \\
\hline Treonina (\%) & 0,75 \\
\hline Triptófano (\%) & 0,24 \\
\hline Relación Arg:Lys & 1,11 \\
\hline Ca (\%) & 0,95 \\
\hline P disponible (\%) & 0,42 \\
\hline
\end{tabular}

\section{RESULTADOS Y DISCUSIÓN}

No hubo efecto $(p>0,05)$ del factor línea sobre ninguna de las de las variables hematológicas y productivas evaluadas (Tabla 2). Sin embargo, se observó una interacción $(p<0,05)$ del factor ambiental, sobre el factor línea de pollo. Independientemente del linaje, las aves que estuvieron bajo el efecto del estrés calórico crónico presentaron $(p<0,05)$ los mayores valores para el hematocrito, concentración de hemoglobina, número de leucocitos totales, linfocitos, monocitos y porcentaje de mortalidad; y del mismo modo fueron los que estadísticamente $(p<0,05)$ mostraron valores inferiores para la relación heterófilo/linfocito, ganancia de peso, consumo de alimento y peor conversión, respecto a los pollos criados bajo la temperatura ambiental del Piedemonte Amazónico (Tabla 3). Ninguna media de tratamientos difirió $(p>0,05)$ de las demás al ser evaluadas las variables: número de eosinófilos y basófilos (Tabla 3). Los resultados permiten inferir que, independientemente de la línea comercial de pollo, la respuesta hematológica y productiva de las aves sigue un comportamiento similar cuando se exponen a condiciones de estrés calórico crónico. En las Tablas 2 y 3 las medias de tratamientos de una misma variable seguidas por letras minúsculas diferentes, dentro de la misma línea, son estadísticamente diferentes entre sí por la prueba de $F(p<0,05)$. 


\section{Condición estrés calórico crónico inducido}

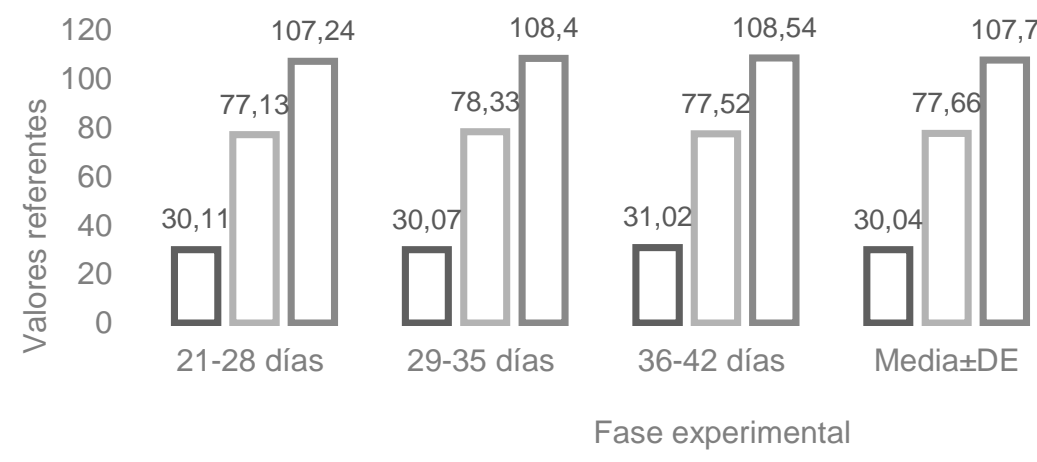

Temperatura $\left(\mathrm{C}^{\circ}\right) \quad$ 口Humedad relativa $(\%) \quad$ andice de confort termico $(\mathrm{T}+\mathrm{HR})$

Fig. 1: Valores promedios del periodo experimental de temperatura, humedad relativa ambiental e índice de confort térmico, bajo ambiente controlado en el galpón

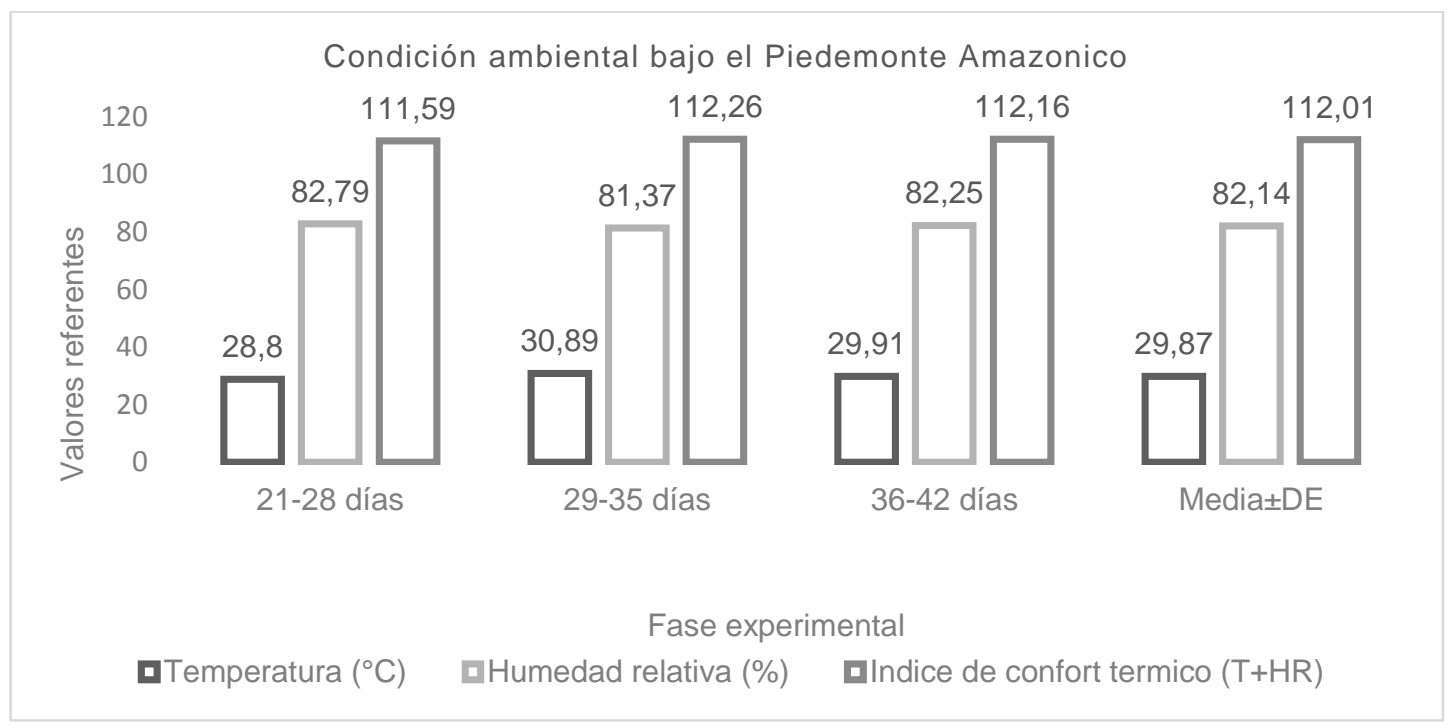

Fig. 2: Valores promedios del periodo experimental de temperatura, humedad relativa ambiental e índice de confort térmico, bajo condición ambiental del galpón bajo el Piedemonte Amazónico colombiano

Hematocrito. Se observó una diferencia $(p<0,04)$ al evaluar la interacción del factor estrés calórico crónico sobre el hematocrito. Los promedios máximos de hematocrito $(32,97 \%)$ obtenidos, independiente del linaje de las aves bajo estrés calórico crónico, se explican como consecuencia del efecto de la intensidad y duración del estímulo estresor (Puvadolpirod y Thaxton, 2000); ocasionado por las altas temperaturas y humedades, con índices de confort térmico $>70$ (Figura 1), en donde un incremento de la actividad muscular, acompañado de la movilización de plasma a los músculos puede aumentar la eritropoyesis y el hematocrito como respuesta a la carencia de oxígeno en los tejidos, debido a la hipoxemia (Olkowski et al., 2005); mientras el índice inferior de hematocrito $(29,20 \%)$ encontrado en los pollos expuestos a las variables temperaturas y humedades ambientales diurnas y nocturnas del Piedemonte Amazónico (Figura 2.), se explica probablemente a que las aves tuvieron la oportunidad de una recuperación homeostática en las horas más frescas de la noche, con posterior retorno a valores más bajos.

No obstante los resultados, todos los valores de hematocrito obtenidos en esta investigación, se encuentran dentro de los valores de referencia normal (22 a 35\%) para pollos de engorde macho a los 42 días de edad (Talebi et al., 2005), lo cual permite inferir, que no hubo pérdida excesiva de fluidos corporales; garantizando un volumen mínimo de glóbulos rojos circulantes, con transporte suficiente de oxígeno a las células. Laganá (2007) tampoco encontró alteración en los porcentajes del hematocrito en pollos de engorde criados desde los 21 a 42 días de edad bajo condiciones de estrés calórico crónico. 
Tabla 2: Promedios $( \pm \mathrm{DE})$ de variables hematológicas y zootécnicas de dos líneas de pollos de engorde a los 42 días de edad sometidos a las condiciones ambientales experimentales

\begin{tabular}{|c|c|c|}
\hline \multirow[b]{2}{*}{ Parámetro } & \multicolumn{2}{|c|}{$\begin{array}{l}\text { Condiciones ambientales experimentales en } \\
\text { los galpones }\end{array}$} \\
\hline & Línea $\mathrm{A}$ & Línea B \\
\hline Hematocrito (\%) & $30,62 \pm 1,74 \mathrm{a}$ & $31,55 \pm 1,91 \mathrm{a}$ \\
\hline Hemoglobina $(\mathrm{g} / \mathrm{dL})$ & $9,18 \pm 1,43 \mathrm{a}$ & $9,26 \pm 1,54 \mathrm{a}$ \\
\hline Leucocitos totales $\left(\mathrm{mm}^{3}\right)$ & $23285,44 a$ & $23530,66 \mathrm{a}$ \\
\hline Linfocitos (\%) & $54,40 \mathrm{a}$ & $54,98 \mathrm{a}$ \\
\hline Eosinofilos (\%) & $1,025 \mathrm{a}$ & $1,025 \mathrm{a}$ \\
\hline Basófilos (\%) & $0,7 \mathrm{a}$ & $0,7 \mathrm{a}$ \\
\hline Monocitos (\%) & $1,035 \mathrm{a}$ & $1,065 \mathrm{a}$ \\
\hline $\begin{array}{l}\text { Relación } \\
\text { heterófilo/linfocito }\end{array}$ & $0,914 \mathrm{a}$ & $0,919 \mathrm{a}$ \\
\hline Ganancia de peso (g) & $1272,43 \pm 233,1 \mathrm{a}$ & $1217,835 \pm 234,55 \mathrm{a}$ \\
\hline Consumo de alimento $(\mathrm{g})$ & $2683,6 \pm 561,15 \mathrm{a}$ & $2637,9 \pm 537 \mathrm{a}$ \\
\hline Conversión alimenticia (g) & $2140,03 \pm 397,13 \mathrm{a}$ & $2184,9 \pm 385,8 \mathrm{a}$ \\
\hline Mortalidad (\%) & $8,25 \pm 2,46 \mathrm{a}$ & $8,25 \pm 2,42 \mathrm{a}$ \\
\hline ANAVAR $(P)$ & ns & ns \\
\hline
\end{tabular}

Tabla 3: Promedios ( \pm DE) de variables hematológicas y zootécnicas de pollos de engorde a los 42 días de edad sometidos a estrés calórico crónico y condición ambiental en el Piedemonte Amazónico colombiano

\begin{tabular}{|c|c|c|}
\hline \multirow{2}{*}{ Variables } & \multicolumn{2}{|c|}{ Condición ambiental en el galpón } \\
\hline & Estrés calórico crónico & Ambiental del Piedemonte Amazónico \\
\hline Hematocrito (\%) & $32,97 \pm 2,0 a$ & $29,20 \pm 1,65 b$ \\
\hline Hemoglobina $(\mathrm{g} / \mathrm{dL})$ & $10,71 \pm 1,45 a$ & $7,73 \pm 1,52 b$ \\
\hline Leucocitos totales $\left(\mathrm{mm}^{3}\right)$ & $24274,6 a$ & $22541,42 b$ \\
\hline Linfocitos (\%) & $74,16 a$ & $35,22 b$ \\
\hline Eosinofilos (\%) & $1,035 a$ & $1,015 a$ \\
\hline Basófilos (\%) & $0,72 a$ & $0,70 \mathrm{a}$ \\
\hline Monocitos (\%) & $1,28 a$ & $0,82 b$ \\
\hline Relación heterófilo/linfocito & $0,369 \mathrm{~b}$ & $1,464 a$ \\
\hline Ganancia de peso (g) & $1062,05 \pm 225,35 b$ & $1428,21 \pm 242,3 a$ \\
\hline Consumo de alimento (g) & $2475,10 \pm 581,71 b$ & $2846,4 \pm 516,4 a$ \\
\hline Conversión alimenticia (g) & $2330,49 \pm 403,53 b$ & $1992,98 \pm 379,35 a$ \\
\hline Mortalidad (\%) & $13 \pm 3,2 a$ & $3,5 \pm 1,675 b$ \\
\hline
\end{tabular}

Hemoglobina. El mayor valor de hemoglobina presentado por las aves bajo condiciones de estrés calórico crónico, comparado con las criadas bajo las condiciones ambientales del Piedemonte Amazónico coincide con los reportes de Furlan et al., (1999), quienes detectaron incremento $(p<0,05)$ en la concentración de hemoglobina en pollos de engorde sometidos a estrés por calor. Se evidencio una alta $(p<0,039)$ interacción del factor estrés calórico crónico sobre la variable hemoglobina. Este hallazgo se relaciona con el incremento de la actividad metabólica necesaria para satisfacer las demandas energéticas, tanto para mantenimiento así 
como para crecimiento, bajo condiciones extremadamente estresantes y como consecuencia de una mayor demanda de oxígeno, al poner en marcha al máximo y en forma continua los mecanismos fisiológicos de termorregulación bajo esta forma de estrés. Sin embargo, los valores obtenidos para cada línea genética se encuentran dentro los valores fisiológicos normales de concentración de hemoglobina (7 - 13 g/dL) en pollos de engorde machos a los 42 días de edad (Talebi et al., 2005). Resultados divergentes, a los presentes, fueron observados por Nowaczewski y Kontecka (2012), quienes no encontraron diferencia estadística ( $p>0,05)$ en la concentración de hemoglobina entre pollos estresados por calor y aquellos criados bajo temperatura termoneutra.

Leucograma. Los valores de leucocitos totales en las dos líneas de pollo encontrados en esta investigación están dentro del intervalo de 12000 a 30000 células $/ \mathrm{mm}^{3}$ considerado fisiológicamente normal (Talebi et al., 2005). La variabilidad en el número de leucocitos totales, entre las aves expuestas a las diferentes condiciones de temperatura (Tabla 3), se explica en función de una mayor liberación de corticosterona durante el estrés crónico (López et al., 2014); (Mumma et al., 2006). Según Puvadolpirod y Thaxton (2000) el aumento de la concentración de corticosterona sanguínea, conduce a una tendencia en el incremento de leucocitos totales, denominado como leucitosis fisiológica, situación que se pudo presentar bajo la condición experimental de estrés calórico crónico, en donde un incremento en las concentraciones de glucocorticoides endógenos aumentarían, subsecuentemente, el número de leucocitos totales durante el tiempo que permaneció el estímulo estresor.

Relación Heterofilo/Linfocito $(H / L)$. La mayor relación $(\mathrm{H} / \mathrm{L})$ observada en las aves expuestas a la temperatura ambiental del Piedemonte Amazónico (Tabla 3), se asocia con cuadros de estrés agudo, en donde la temperatura afecto intensamente durante periodos transitorios de tiempo. Se evidencio una alta $(p<0,034)$ interacción del factor temperatura ambiental del Piedemonte Amazónico sobre esta relación. La relación (H/L) calculada se elucida por la presencia de heterofilia y linfopenia, comportamiento celular fisiológico que coincide con la respuesta en la fase primaria de las aves ante el estrés (Prieto y Campo, 2010), explicado como parte del efecto estresor agudo ocasionado por las condiciones ambientales variables del Piedemonte Amazónico, y probablemente por resultado directo del incremento repentino de la concentración de glucocorticoides, los cuales provocaron afluencia de heterófilos desde la medula ósea, atenuando la salida de estos hacia otros espacios corporales. Contrario a esto, los linfocitos se adhieren a los endotelios, afectando su tránsito hasta los tejidos linfoides (Prieto y Campo, 2010). Los resultados obtenidos ratifican los reportes de Laganá et al., (2005) quienes hallaron incremento en la relación heterófilo/linfocito por encima de 0,91 (Furlan et al., 1999), explicado por una reducción en la cantidad de linfocitos circulantes debido a una mayor concentración de corticosterona. La menor relación $\mathrm{H} / \mathrm{L}$ revelada bajo estrés calórico crónico (Tabla 3), se encuentra próxima a los valores normales de referencia para pollos de engorde macho a los 42 días de edad (Tabeli et al., 2005); explicándose como una réplica adaptativa celular a este tipo de estrés durante la respuesta bifásica secundaria (Prieto y Campo, 2010), pudiendo indicar resistencia inmune ante procesos de estrés crónico.

Leucograma diferencial. Los resultados del leucograma diferencial, coinciden con los obtenidos por Laganá (2007) quien tampoco noto cambios en el conteo de estas células en pollos de engorde sometidos a estrés calórico crónico. En contraste, Cïrule et al., (2012) reportaron eosinopenia y basopenia en aves bajo estrés calórico. A pesar de no estar totalmente identificada la función exacta de los eosinófilos en las aves, existe evidencia de que estas células participan en la fase inicial de la respuesta al estrés agudo en pollos de engorde. Los resultados divergentes obtenidos respecto a la literatura existente se pueden deber, en parte, a diferencias metodológicas; sin embargo, además de la temperatura ambiente elevada, las diferencias en la magnitud, duración y tipo de estrés al que se sometieron las aves son factores importantes que se deben tener en cuenta para analizar la respuesta. Por otra parte, se observa un recuento inferior de monocitos en los pollos bajo temperatura ambiental del Piedemonte Amazónico, contrastado con los animales sometidos a estrés calórico crónico (Tabla 3), evidenciándose en estos una diferencia mayor de 74,80\% en el número de monocitos, en respuesta a una presunta mejor adaptación ante esta forma de estrés. El recuento más bajo de monocitos hallado en las aves bajo la temperatura ambiental del Piedemonte Amazónico se puede explicar por el efecto directo quimiotáctico de la hormona adrenocorticotropina sobre estas células, evento que ocasiona cambios en los componentes leucocitarios circulantes, permitiendo inferir una baja respuesta fagocitara ante diferentes microorganismos, dentro de los estados de estrés agudo (Lam, 1999).

Ganancia de peso. Se observó una alta interacción $(p<0,03)$ del factor estrés calórico crónico sobre esta variable, notándose un efecto de pérdida de $25,61 \%$ en la ganancia de peso de las líneas bajo la temperatura estrés calórico crónico, respecto a la condición ambiental del Piedemonte Amazónico (Tabla 3). Estudios similares reportan pérdidas en la ganancia de peso por estrés calórico crónico hasta de $74 \%$ (Dozier et al., 2005). El mejor rendimiento en cada linaje, fue favorecido por la variabilidad de la temperatura ambiental del Piedemonte Amazónico, relacionado con los periodos de termoneutralidad diarios, permitiendo una recuperación parcial de las aves al maximizar el uso de la energía, mejorando el desempeño productivo. 
Una digestión ineficiente del alimento con reducción en el consumo de alimento y de algunos nutrientes, son los agravantes del regular comportamiento de este índice. Los altos niveles de corticosterona (López et al., 2014) con el subsecuente incremento del catabolismo de las proteínas (Mumma et al., 2006) y el mayor gasto de energía utilizada durante la contracción muscular, asociada a la pérdida de calor latente mediante el enfriamiento evaporativo, aumentan la sensibilidad metabólica con detrimento productivo, frente a condiciones ambientales no óptimas (Baracho et al., 2011).

Aunque la ganancia de peso obtenida en los animales bajo la condición ambiental del Piedemonte Amazónico (Tabla 3) fue mejor que la de aquellos bajo estrés calórico crónico, estos resultados son inferiores al ser comparados con el peso referente (línea A: 1,957 g; línea B: 1,947 g) expuesto en la guías de rendimiento productivo (Cobb-Vantress, 2014); (Aviagen, 2014), con una diferencia biológica negativa de $24,7 \%$ para la línea A y $28,8 \%$ para la línea B. El bajo desempeño, se explica por el efecto negativo de la condición ambiental ocasionado por la alta temperatura y humedad, con registro de una sensación térmica $>70$ (Figura 2.), la cual interfirió con la eliminación del mayor calor metabólico originado por las aves, en esta fase final de crecimiento (Cobb-Vantress, 2014).

Consumo de alimento. Se observó una interacción $(p<0,04)$ del factor ambiental Piedemonte Amazónico sobre el consumo de alimento; en donde las oscilaciones de temperatura diurna y nocturna favorecieron un mayor consumo de alimento, explicado por la adaptación de las aves, a comer más en las horas más frescas del día y menos durante las horas críticas del pico de calor. Sin embargo, al confrontar las variables productivas en cada linaje bajo las condiciones ambientales del Piedemonte Amazónico (Tabla 3), con el consumo de alimento acumulado objetivo según el linaje (línea A: 3,727 g y línea B: 3,673 g) expuestos en la guías de rendimiento productivo (Cobb-Vantress, 2014); (Aviagen, 2014); se infiriere que las líneas A y B tuvieron, respectivamente, $23,3 \%$ y $22,7 \%$ menor consumo de alimento, frente al referente para estas dos líneas de pollo criadas bajo condiciones constantes de termo neutralidad. Resultados similares reporto Abu-Dieyeh (2006) al calcular una disminución en el consumo de alimento del 22\%, en pollos desde 21 a 42 días de dad bajo estrés calórico a $35^{\circ} \mathrm{C}$. La primera respuesta del ave al estrés por calor, es la disminución del apetito y del consumo de alimento (Abu-Dieyeh, 2006a), mecanismo usado para reducir la carga calórica endógena, dentro de los ajustes fisiológicos necesarios para el mantenimiento de la homeostasis térmica (Abu-Dieyeh, 2006b). Sin embargo, implica también una baja ingesta de nutrientes esenciales para la producción y bienestar (Ferreira et al., 2011). Los resultados obtenidos permiten sugerir que el nivel, la duración y el tipo de estrés, son factores directos que afectan esta variable zootécnica.

Mortalidad. Se observó una divergencia superior al 73,08\% en la mortalidad de los animales bajo la condición ambiental de estrés calórico crónico, respecto a la condición ambiental del Piedemonte Amazónico (Tabla 3). Se evidencio una alta interacción $(p<0,03)$ del factor estrés calórico crónico sobre la mortalidad. La tasa de mortalidad calculada muestra una diferencia de $44,12 \%$ y $47,77 \%$, respectivamente, para las líneas A y B bajo la temperatura ambiental del Piedemonte Amazónico, en contraste con el índice zootécnico de referencia para aves de las dos líneas (A: 1,90\%; B: 1,88\%) bajo condiciones ambientales de neutralidad térmica (CobbVantress, 2014); (Aviagen, 2014). Los resultados encontrados coinciden con los de Laganá (2007), quien encontró $8,63 \%$ de mortalidad en pollos de engorde después de someterlos a estrés calórico crónico con temperatura de $32^{\circ} \mathrm{C}$, desde los 21 a 42 días de edad.

La mortalidad obtenida en este trabajo se explica parcialmente por el grado de confinamiento experimental, interfiriendo con la elaboración de los ajustes comportamentales necesarios para el mantenimiento de la homeostasis térmica, donde las aves buscaban mantenerse sanas y vivas (López et al., 2014). El ineficiente enfriamiento evaporativo de los pollos, a la postre, generó almacenamiento continuo de calor, con incremento de la temperatura corporal superior a los $42^{\circ} \mathrm{C}$, conduciendo a las aves a un nivel letal mediante el agotamiento y postración. La muerte en estos casos, generalmente, es ocasionada por falla cardiovascular acompañada de un desbalance iónico sanguíneo.

\section{CONCLUSIONES}

El cuadro hemático y las variables productivas evaluadas mostraron variaciones detrimentales importantes, en cada una de las dos líneas de aves, como efecto del estrés calórico crónico; no obstante, en este mismo sentido el número de eosinófilos y basófilos no reflejo diferencias contundentes. La relación heterófilo/linfocito expresada por las dos líneas bajo la temperatura estrés calórico crónico, estuvo más próxima a los valores normales de referencia, probablemente, como consecuencia de un incipiente proceso de adaptación a este tipo de estrés.

Las dos líneas de pollo evaluadas, fueron afectadas de forma similar por el estrés calórico ambiental, sin evidencia de una ventaja en la resistencia a este tipo de estrés de una línea sobre la otra. 
La producción intensiva de pollo de engorde en la región del Piedemonte Amazónico colombiano, no se ajusta a los patrones zootécnicos de producción de las regiones con temperaturas de climas templados, razón por la cual, además de adaptar todos los recursos tecnológicos para minimizar el efecto del estrés calórico en las aves, se hace necesario establecer las curvas referentes de producción y rendimiento específicas para la región, cuando no se dispone de galpones de ambiente controlado.

\section{REFERENCIAS}

Abbas, A. O., A. K. A. El-Dein, A. Desoky, e M. A. A. Galal. The effects of photoperiod programs on broiler chicken performance and immune response. Int. J. Poult. Sci.: 7, pp. 665-671 (2008)

Abu-Dieyeh, Z.H.M., Effect of chronic heat stress and longterm feed restriction on broiler performance. Int. J. Poult. Sci.: 5, pp. 185-190 (2006a)

Abu-Dieyeh, Z.H.M., Effect of high temperature per se on growth performance of broilers. International Journal of Poultry Science: 5, pp. 19-21 (2006b)

Almeida, P.F., Salles, José A.A., Farias, T.M.B., y Curvelo Santana, J.C., Aprovechamiento de Patas de Pollos como Alternativa para disminuir Residuos Generados en los Mataderos, doi: 10.4067/S071807642012000400006, Inf. Tecnol. (en línea), 23(4), 45-52 (2012)

Amaral A.G., Yanagi Junior T., Lima R.R., Teixeira V.H., Schiassi L., Efeito do ambiente de produção sobre frangos de corte sexados criados em galpão comercial. Arquivo Brasileiro de Medicina Veterinária e Zootecnia: (63), pp. 649-658. (2011)

Aviagen, R., Objetivos de rendimiento y especificaciones nutricionales. Huntsville, Alabama. USA., pp. 3 -13 (2014)

Baracho M.S., Nääs I.A., Nascimento G.R., Cassiano J.A., Oliveira K.R., Surface temperature distribution in broiler houses. Brazilian Journal of Poultry Science.13, pp.177-182 (2011)

Briones, N., T. Jiménez y M. Farías. Evaluación de espectrofotómetros para la elaboración de material de referencia empleado en la calibración y el control de la determinación de hemoglobina. Revista de la Facultad de Medicina, ISSN: 0798-0469 (en línea), 32(1), 46-53, 2009. http://www.scielo. org.ve/scielo. htm. Acceso: 22 de enero (2016).

Cahaner A., Ajuh J.A., SiegmundSchultze M., Azoulay Y., Druyan S., Valle Zárate A., Effect of the genetically reduced feather coverage in naked neck and featherless broilers on their performance under hot conditions. Poultry Science: 87(12), pp. 2517-2527 (2008)

Cïrule, D.; Krama, T.; Vrublevska, J.; Rantala, M.J.; Krams, I., A rapid effect of handling on counts of white blood cells in a wintering passerine bird: a more practical measure of stress? Journal of Ornithology, Heidelberg: 153, pp. 161-166 (2012)

Cobb-Vantress, Inc. Suplemento informativo de rendimiento y nutrición del pollo de engorde Cobb® 500 . Siloam Springs, Arkansas, USA, p. 6 (2014)

Dozier, W. A., B. D. Lott, y S. Branton. Growth responses of male broilers subjected to increasing air velocities at high ambient temperatures and a high dew point. Poult. Sci.: 84, pp. 962-966 (2005)

Farfán, C., Oliveros, Y., e De Basilio, V., Efecto de la adición de minerales en agua o en alimento sobre variables productivas y fisiológicas en pollos de engorde bajo estrés calórico. Zootecnia Tropical, ISSN: 07987269 (en línea), 28(3) ,363-373,2010. http://www.scielo.org.ve/scielo. htm. Acceso: 22 de enero (2016)

Ferreira VMOS, F.N.S., Belloni M., Aguirre G.M.Z., Caldara F.R., Nääs I.A., Infrared thermography applied to the evaluation of metabolic heat loss of chicks fed with different energy densities. Brazilian Journal of Poultry Science, 3, pp.113-118. (2011)

Furlan, R.L., Faria, Filho, D.E., Rosa, P.S. y Macari, M., Does low-protein diet improve broiler performance under heat stress conditions?. Rev. Bras. Cienc. Avic. (online),Vol.6, n.2, pp. 71-79. ISSN 18069061. http://dx.doi.org/10.1590/S1516-635X2004000200001 (2004)

Furlan R.L., Macari M, Moraes V.B.M., Malheiros R.D., Malheiros E.B., Secato E.R., Alterações hematológicas e gasométricas em diferentes linhagens de frangos de corte submetidos ao estresse calórico agudo. Rev. Bras. Cien. Avic. 1, pp. 77-84 (1999)

Holdridge, L. R., Ecología basada en zonas de vida. IICA. Serie de libros y materiales educativos. No. 34 (1978) 
Laganá, C., Níveis dietéticos de proteína e gordura e parâmetros bioquímicos, hematológicos e empenamento em frangos de corte estressados pelo calor. R. Bras. Zootec: 36(6), pp.1783-1790 (2007)

Laganá, C., A. M. L. Ribeiro, F. H. D. González, L. L. de Almeida, S. L. Terra, and T. R. Barbosa. Suplementação de vitaminas e minerais orgânicos nos parâmetros bioquímicos e hematológicos de frangos de corte em estresse por calor. Bol. Ind. Anim.: 62, pp.157-165 (2005)

Lam, K. M. Studies on chicken peripheral blood monocytes. Comparative Haematology Internacional: 9, pp. 142-147 (1999)

López, E. A. D., Velásquez, L. F. U., y Solarte, W. N. Bioquímica sanguínea y concentración plasmática de corticosterona en pollo de engorde bajo estrés calórico. Revista de Medicina Veterinaria: 28, pp. 31-42 (2014)

Lu, Q. P., J. Wen, and H. F. Zhang. Effect of chronic heat exposure on fat deposition and meat quality in two genetic types of chicken. Poult. Sci.: 86, pp. 1059-1064 (2007)

Mitchell, E. B.; Johns, J. Avian hematology and related disorders. Veterinary Clinics of North America: Exotic Animal Practice: 11, pp. 501-522 (2008)

Mumma, J. O., Thaxton, J. P., Vizzier-Thaxton, Y., y Dodson, W. L. Physiological stress in laying hens. Poultry science: 85(4), pp. 761-769 (2006)

National Research Council (NRC). Nutrient requirements of poultry. 9a Rev. Ed. National Academy Press. Washington, D.C. (1994)

Natt, M.P. y C.A. Herrick. The new diluent for counting erythrocyte and leukocytes of the chicken. Poult Sci.: 31, pp. 735-738 (1952)

Nowaczewski, S. y Kontecka, H. Haematological indices, size of erythrocytes and haemoglobin saturation in broiler chickens kept in commercial conditions. Animal Science Papers and Reports: 30 (2) pp. 181-190 (2012)

Olkowski, A. A., T. Duke, y C. Wojnarowicz. The aetiology of hypoxaemia in chickens selected for rapid growth. Comp. Biochem. Physiol: A (141), pp. 122-131 (2005)

Puvadolpirod S., Thaxton J.P., Model of Physiological Stress in Chickens 1. Response Parameters. Poult Sci: 79(4), pp. 363-369 (2000)

Prieto, M. T., y Campo, J. L., Effect of heat and several additives related to stress levels on fluctuating asymmetry, heterophil: lymphocyte ratio, and tonic immobility duration in White Leghorn chicks. Poultry science:: 89(10), pp. 2071-2077 (2010)

Rostagno H.S., Albino L.F.T., e Donzele J.L., Tabelas brasileiras para aves e suínos: composição de alimentos e exigências nutricionais. $3^{\text {a }}$ Ed. Viçosa, Brasil: UFV. Departamento de Zootecnia, (2011)

Statistical Analysis System Institute. SAS/STAT. User's Guide (Versión 8, 2a ed.) Cary, NC. (2007)

Silva, J. E., Thermogeneic mechanisms and their hormonal regulation. Physiological Rev. 86, pp. 435-464 (2006)

Talebi, A.; Asri-rezaei, S.; Rozeh-chai, R.; Sahraei, R. Comparative studies on haematological values of broiler strains (Ross, Cobb, Arbor-acres and Arian). International Journal of Poultry Science: 4 (8), pp. 573-57 (2005)

Voslarova, E., P. Chloupek, I. Bedanova, P. Suchy, V. Pistekova, y V. Vecerek. The effect of pre-sampling handling time on blood plasma biochemical parameters of broilers. Dtsch. Tierarztl. Wochenschr: 115, pp. 6265 (2008) 